\title{
Research Paper \\ The Relationship of Sleep Quality With Body Mass Index in Male College Students With and Without Regular Exercise
}

\author{
Oveys Gilanian Amiri $^{1} \mathrm{O},{ }^{*}$ Masomeh Habibian ${ }^{1} \mathrm{C}$
}

1. Department of Physical Education and Sports Sciences, Qaemshahar Branch, Islamic Azad University, Qaemshahar, Iran.

\begin{tabular}{|l|l|l|l|l}
\hline $\begin{array}{l}\text { Use your device to scan } \\
\text { and read the article online }\end{array}$ & $\begin{array}{l}\text { Citation Gilanian Amiri O, Habibian M. The Relationship of Sleep Quality With Body Mass Index in Male College Students With } \\
\text { and Without Regular Exercise. The Journal of Qazvin University of Medical Sciences. 2020; 24(1):14-23. https://doi.org/10.32598/ } \\
\text { JQUMS.24.1.2 }\end{array}$ \\
dof https://doi.org/10.32598/JQUMS.24.1.2
\end{tabular}

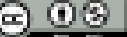

Received: 23 Dec 2018

Accepted: 15 Apr 2019 Available Online: 01 Apr 2020

Keywords:

Body mass index Exercise, Sleep quality

\section{A B S T R A C T}

Background Poor Sleep Quality (SQ) may lead to health problems through an increase in body weight and adipose tissue. Physical activity and or exercise are effective factors in SQ and obesity.

Objective The current study aimed to investigate the relationship of SQ with Body Mass Index (BMI) in male college students with and without regular exercise.

Methods In this descriptive-analytical study, 100 male college students with and without regular exercise were selected using a purposive and convenience sampling method and divided into two groups of active with normal weight $\left(\mathrm{n}=50, \mathrm{BMl}=21.9 \pm 1.2 \mathrm{~kg} / \mathrm{m}^{2}\right)$ and inactive with overweight $(\mathrm{n}=50, \mathrm{BMI}=28.3 \pm 5.2$ $\mathrm{kg} / \mathrm{m}^{2}$ ) groups. The Pittsburg Sleep Quality Index (PSQI) questionnaire was used to measure SQ, where a total score of more than 5 indicates a poor SQ. The data were analyzed using Spearman correlation and Mann-Whitney $U$ tests.

Findings Sixty percent of the students had a poor SQ. The mean score of SQ in the active group was significantly lower than in the inactive group $(P<0.001)$. Moreover, the results showed a significant correlation between the $S Q$ and $B M I$ in overall $(P<0.001)$ and in the inactive group $(P=0.043)$, but not in the active group.

Conclusion Exercise is an effective factor for improving the SQ. Therefore, physical activity/exercise is recommended as a non-pharmacological therapy to improve SQ in overweight young men.

\section{Extended Abstract}

\section{Introduction}

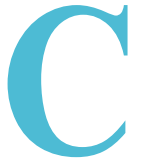

hronic insomnia, as one of the most common sleep disorders, affects a large number of people around the world each year [1]. Sleep disorders occur in a large proportion of the adult population, and it is esti- mated that $10-13 \%$ of adults suffer from chronic insomnia [2]. Short sleep duration is associated with obesity, type 2 diabetes, high blood pressure, and cardiovascular diseases [3]. Feeling tired during the day is a predictor of poor Sleep Quality (SQ), and wakefulness is associated with better SQ [4]. In previous studies, the relationship between short sleep duration and increased Body Mass Index (BMI) [5] and the high correlation of increased BMI with decreased SQ with increased frequency of waking up have also reported [6].

\section{* Corresponding Author:}

Masomeh Habibian

Address: Department of Physical Education and Sports Sciences, Qaemshahar Branch, Islamic Azad University, Qaemshahar, Iran. Tel: +98 (811) 42155025

E-Mail: habibian_m@yahoo.com 
On the other hand, there is a relationship between sleep disorders and weight gain [7]. Insomnia, or its pathophysiological complications, may result in excessive energy intake, leading to increased appetite, excessive consumption of food, and weight gain [8]. Further obesity or increased abdominal fat has been reported in people with shorter sleep duration [9]. Also, excessive consumption of highfat and high-sugar foods and abdominal fat storages may have a calming effect on the brain hyperactivity [2]. Studies have shown that in obese people without sleep disorders or emotional stress, sleep duration is equal to that of normal people, which indicates the importance of diagnosing and treating sleep disorders as a potential therapeutic intervention for obesity [10].

Exercise may be associated with optimal SQ [11, 12], and can improve sleeping efficiency in people with sleep problems. Even after controlling other influencing factors, physical inactivity may be increasingly associated with symptoms of insomnia or poor SQ [13]. The present study aimed to determine and compare the relationship between SQ and BMI in male college students with and without regular physical activities.

\section{Materials and Methods}

The study population in this descriptive study consists of all male college students aged 18-27 years studying at Islamic Azad University of Qaemshahr branch in Iran. Of them, 100 students with and without regular exercise were selected using purposive and convenience sampling methods and divided into two groups of active with normal BMI $(n=50)$ and inactive with overweight $(\mathrm{n}=50)$. The subjects with a previous history of cardiovascular and respiratory diseases, and the use of drugs such as theophylline, aminophylline, corticosteroids and hypnotic tablets were excluded from the study. SQ was measured using a Pittsburgh sleep quality index (PSQI) which evaluates sleep problems during the last 4 weeks. The PSQI has 7 subscales shown in Table 1.

Its total score ranges from 0 to 21 where the score $>5$ indicates poor SQ. The validity and reliability of this questionnaire has been reported in the earlier studies [1]. In the present study, the Cronbach's alpha coefficient for its Persian version was obtained 0.716. The Kolmogorov-Smirnov and Levene's tests were used to determine the normality of data distribution and the equality of variances, respectively. The

Table 1. Comparison of the mean sleep quality and its dimensions in male students

\begin{tabular}{|c|c|c|c|c|}
\hline Dimensions of Sleep Quality & Groups & Mean \pm SD & $\mathbf{P}^{*}$ & $\mathbf{P} * *$ \\
\hline \multirow{2}{*}{ Quality of sleep } & inactive & $1.12 \pm 0.82$ & $<0.001$ & \multirow{2}{*}{$0.004^{\#}$} \\
\hline & active & $0.77 \pm 0.48$ & $<0.001$ & \\
\hline \multirow{2}{*}{ Lag in starting the sleep } & inactive & $1.50 \pm 0.82$ & $<0.001$ & \multirow{2}{*}{$0.042^{\#}$} \\
\hline & active & $1.12 \pm 0.76$ & $<0.001$ & \\
\hline \multirow{2}{*}{ Sleep duration } & inactive & $1.32 \pm 0.69$ & $<0.001$ & \multirow{2}{*}{$0.006^{\#}$} \\
\hline & active & $0.92 \pm 0.52$ & $<0.001$ & \\
\hline \multirow{2}{*}{ Efficiency of the sleep } & inactive & $0.52 \pm 0.42$ & $<0.001$ & \multirow{2}{*}{$0.035^{\#}$} \\
\hline & active & $0.46 \pm 0.30$ & $<0.001$ & \\
\hline \multirow{2}{*}{ Sleep disorders } & inactive & $0.95 \pm 0.59$ & $<0.001$ & \multirow{2}{*}{$0.036^{\#}$} \\
\hline & active & $0.49 \pm 0.40$ & $<0.001$ & \\
\hline \multirow{2}{*}{ Use of hypnotic drugs } & inactive & $0.70 \pm 0.52$ & $<0.001$ & \multirow{2}{*}{$<0.001^{\#}$} \\
\hline & active & $0.40 \pm 0.20$ & $<0.001$ & \\
\hline \multirow{2}{*}{ Disorders of daily functions } & inactive & $1.90 \pm 0.63$ & $<0.001$ & \multirow{2}{*}{$<0.001^{\#}$} \\
\hline & active & $1.55 \pm 0.62$ & $<0.001$ & \\
\hline \multirow{2}{*}{ Overall score of sleep quality } & inactive & $8.05 \pm 1.12$ & 0.012 & \multirow{2}{*}{$<0.001^{\sharp}$} \\
\hline & active & $5.27 \pm 1.83$ & 0.012 & \\
\hline
\end{tabular}

*Kolmogorov-Smirnov test;

** Mann-Whitney U test; "Significant difference compared to the inactive group 
Mann-Whitney U and Spearman correlation tests were used for data analysis. The significance level was set at $\mathrm{P}<0.05$.

\section{Results}

The Mean \pm SD of height, weight and BMI of students in the active group were $176.8 \pm 6.9 \mathrm{~cm}, 65.6 \pm 8.0 \mathrm{~kg}$ and $21.9 \pm 2.1 \mathrm{~kg} / \mathrm{m}^{2}$, respectively, while for those in the inactive group were $169.4 \pm 16.3 \mathrm{~cm}, 85.5 \pm 8.4 \mathrm{~kg}$ and $28.5 \pm 3.2$ $\mathrm{kg} / \mathrm{m}^{2}$, respectively. The mean score of PSQI and its seven dimensions for active subjects was significantly lower than that of inactive subjects $(\mathrm{P}<0.05)$, which indicates that active boys with normal BMI have better SQ and less disturbances compared to the overweight inactive boys (Table1).

In overall, the BMI of subjects had a direct and significant correlation with overall SQ, sleep disturbances, sleeping medication, and daytime dysfunction. However, the BMI of active group had no significant relationship with SQ score and its dimensions; only the BMI of inactive group had a direct and significant relationship with SQ score.

\section{Conclusion}

Findings of the present study indicated that the SQ and its dimensions in the active group were lower compared to those of the inactive group. As a result, it can be said that active men have better SQ than inactive men. Physical activity is associated with improvement in SQ by increasing the non-rapid eye movement sleep (low heart rate and cerebral metabolism are significantly reduced), decreasing rapid eye movement sleep and reducing rapid eye movement latency (the time from the sleep onset to the first epoch of rapid eye movement sleep). Moreover, biological and biochemical changes caused by exercise and physical activity can lead to improved physical/mental health and, consequently, the improvement of SQ. Exercise can regulate some intervening factors such as growth regulating hormone, interleukin-1, prostaglandin D2, adenosine, tumor necrosis factoralfa, prolactin, corticotrophin, vasoactive intestinal peptide; therefore, it can develop the quality of sleep in active individuals $[1,11,13]$. Sports activities can be considered as an effective factor in improving the quality of sleep. Therefore, the use of physical activity or exercise as a non-pharmacological treatment is recommended to improve the quality of sleep in overweight young men.

\section{Ethical Considerations}

\section{Compliance with ethical guidelines}

The present paper with dissertation code of 107214049552002 approved in Ghaemshahr Branch,
Islamic Azad University. The participants were informed about the purpose of the research and its implementation stages; they were also assured about the confidentiality of their information

\section{Funding}

This research did not receive any specific grant from any organization.

\section{Authors' contributions}

All authors had a equal contribution in preparing this article.

\section{Conflicts of interest}

The authors declared no conflict of interest.

\section{Acknowledgements}

The authors would like to thank the students participated in the study for their cooperation. 


\title{
تعيين رابطه كيفيت خواب با شاخص توده بدن در يسران دانشجو با و بدون فعاليت ورزشى
}

\author{
'اويس كيلانيان اميرى' ه "معصومه حبيبيان' \\ 1. آكروه تربيت بلنى، واحد قائمشهر، دانشكاه آزاد اسلامى، قائمشهر، ايران.
}

\begin{abstract}
דيكيد

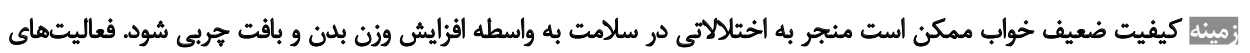

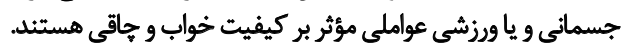

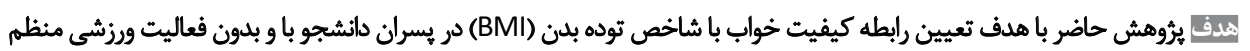
انجام شد.

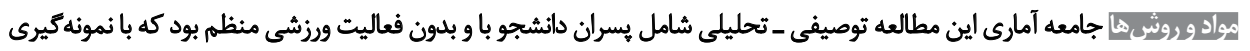

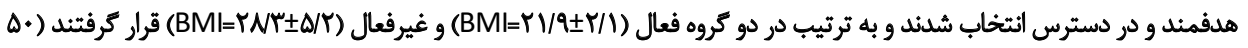

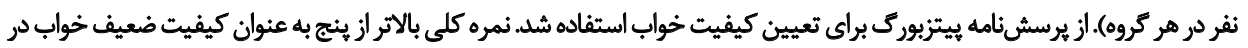

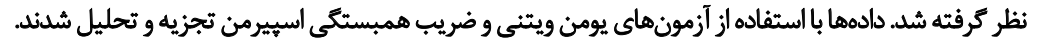

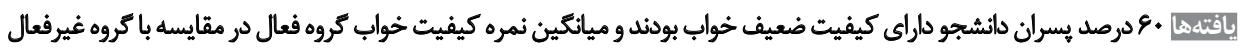

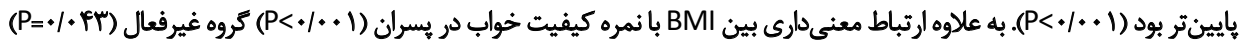

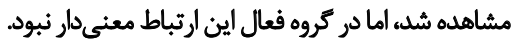

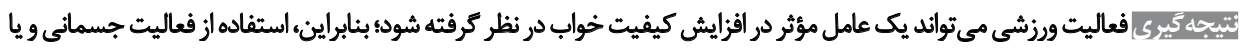

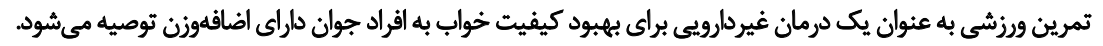

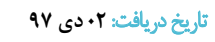

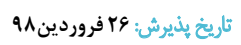
تاريخ اتتشارا: "ا فروردين 1499

كليدوارثها:

شاخص توده بدن، فعاليت ورزشى، كيفيت تودني خواب

خستكى در طول روزء بيشبينى كثنده كيفيت خواب ضعيفتر

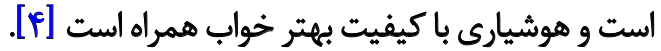

امروزه كنترل دقيق خواب بخش مهابمى از اهداف بالينى است؛

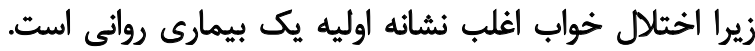

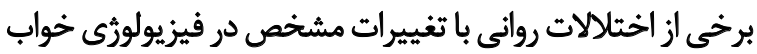

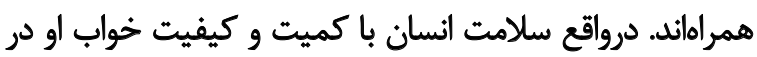

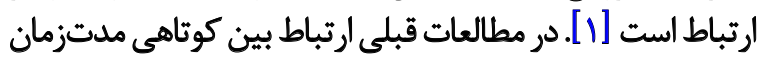

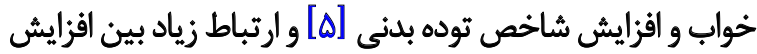

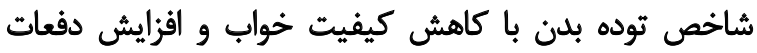

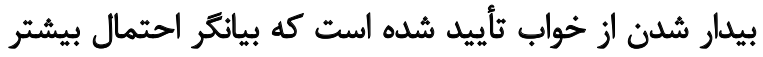

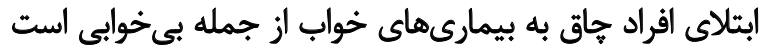

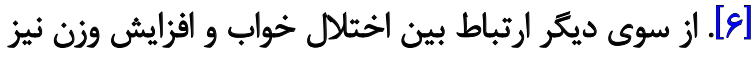

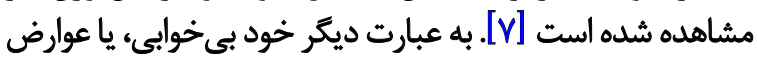

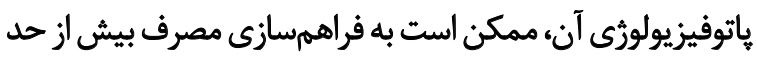

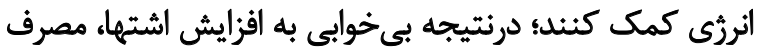

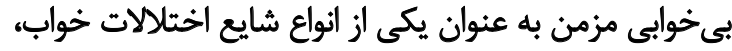

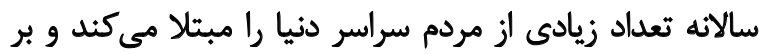

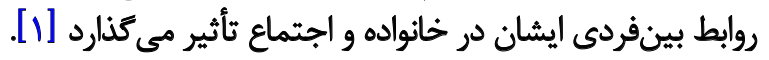

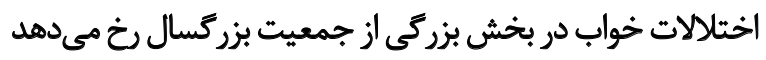

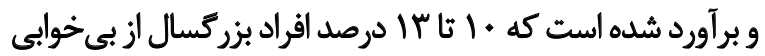

$$
\text { مزمن رنج مىبرند [ب] }
$$

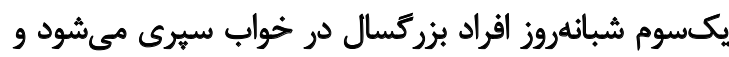

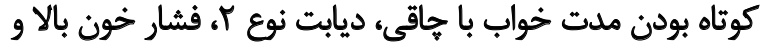

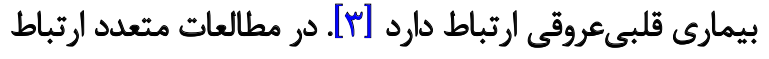

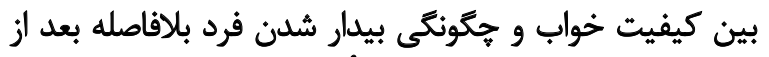

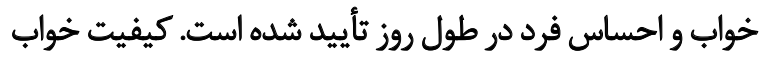

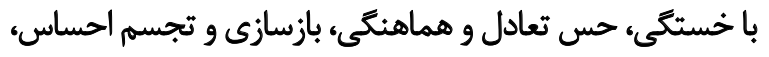
خلقوخو و احساسات جسمانى در بيدارى همراه است. احساس ونس احساس،

$$
\begin{aligned}
& \text { * فويسنده مسيئول: } \\
& \text { معصومه حبيبيان }
\end{aligned}
$$

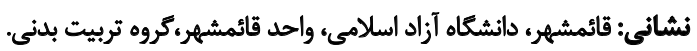

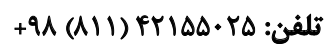

$$
\begin{aligned}
& \text { رايانامه: habibian_m@yahoo.com }
\end{aligned}
$$




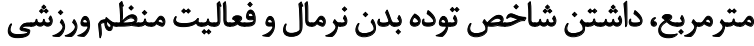

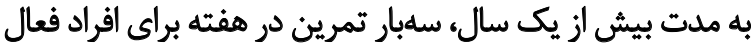

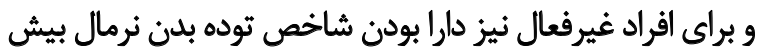

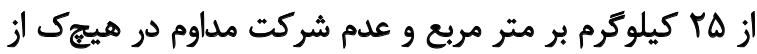

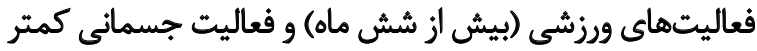

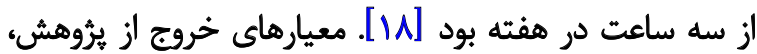

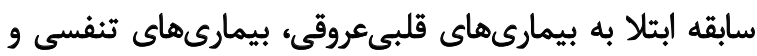

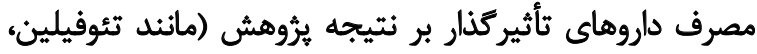

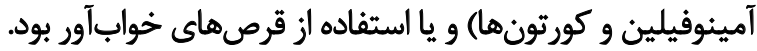

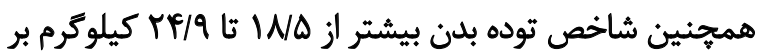

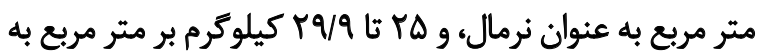
عنوان اضافهوزن در نظر كرفته شد [1]

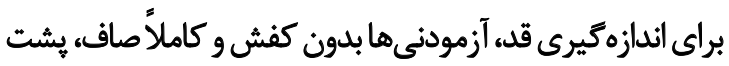

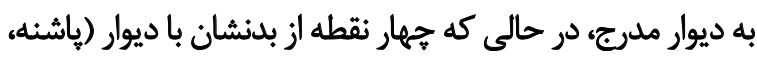

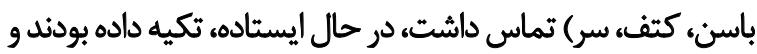

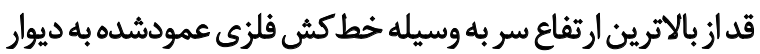

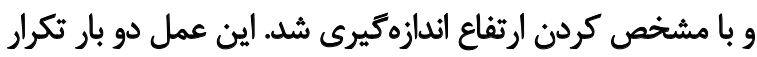

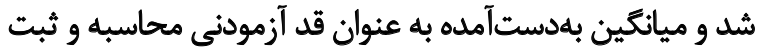

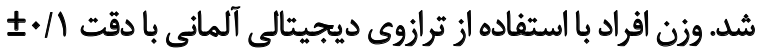

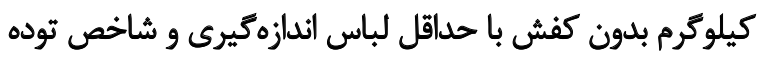

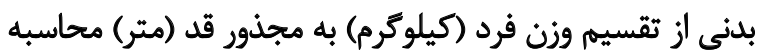

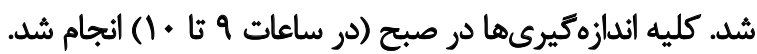

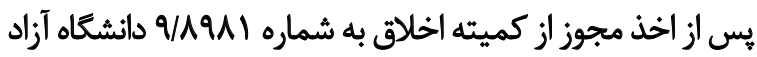
اسلامى واحد قائمشهر، آزمودنى هما به تحقيق اخله راه راه يافتند.

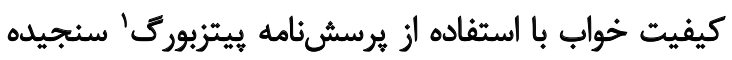

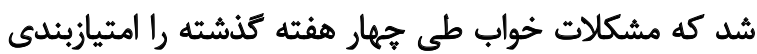

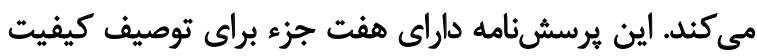

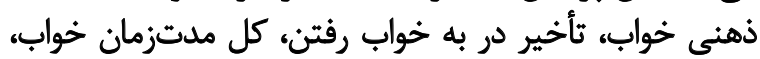

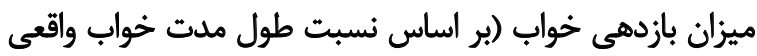

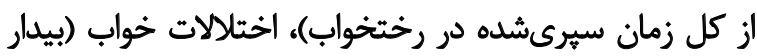

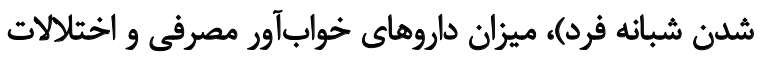

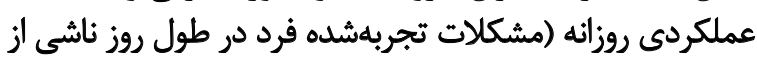

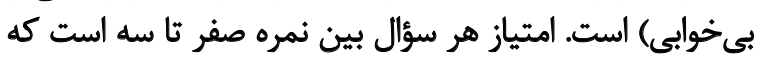

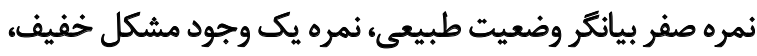

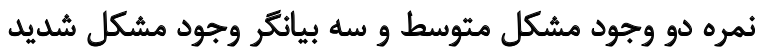

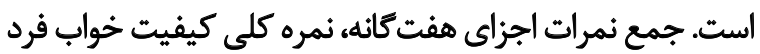

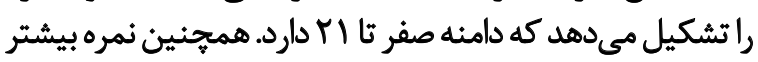

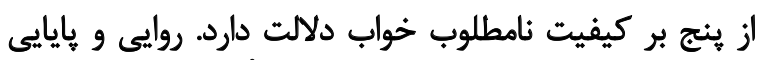

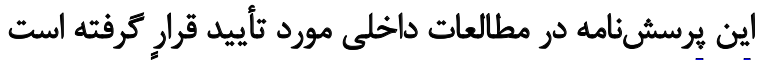

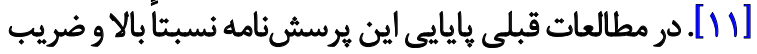

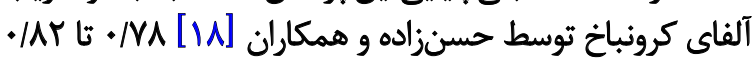

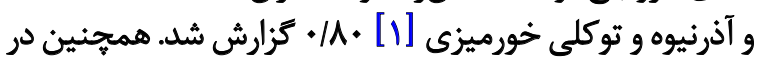

1. Pittsburg sleep quality inde
اضافى غذا و افزايش وزن منجر شود [N]]. علاوه بر اين، احتمال

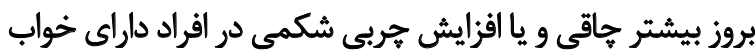

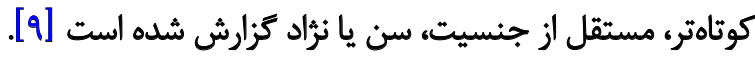

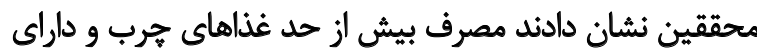

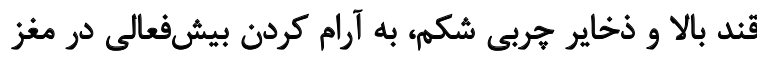

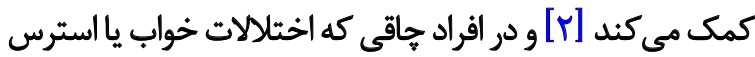

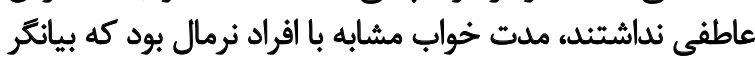

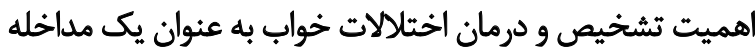

درمانى بالقوه براى جاقي است دمان [.1].

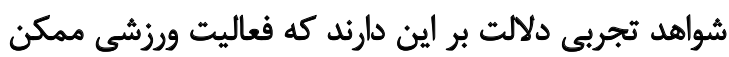

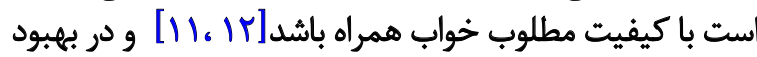

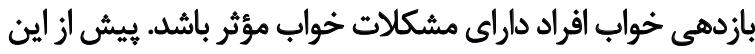

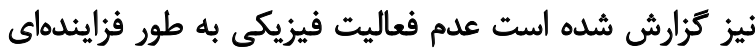

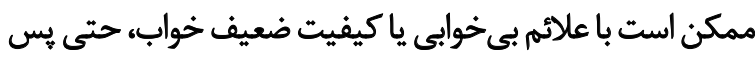

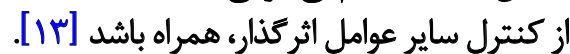

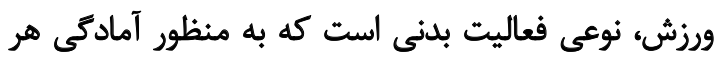

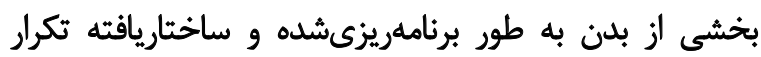

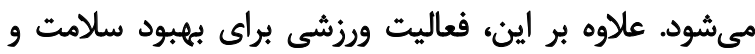

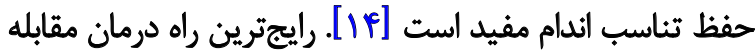

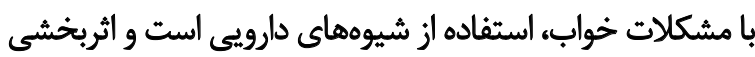

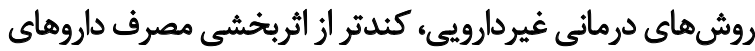

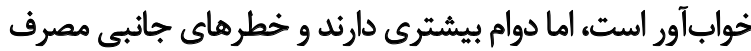

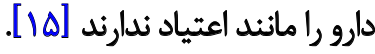

با توجه به نقش مهرم كيفيت خواب و كافي بودن طول مدات

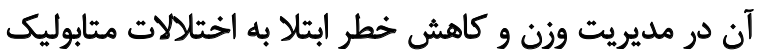

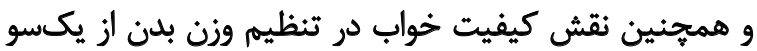

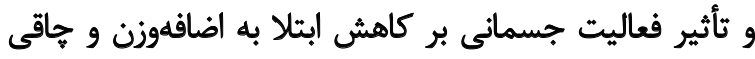

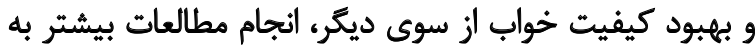

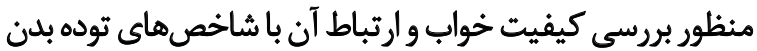

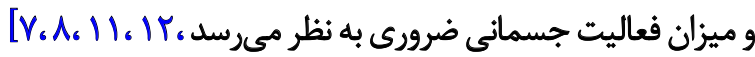

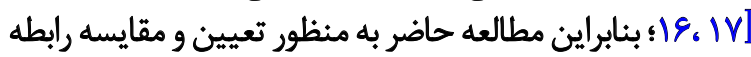

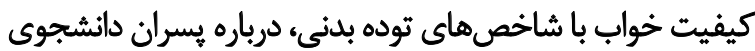
با و بدون فعاليت ورزشى منظم انجام شد.

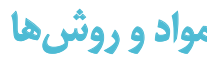

اين مطالعه توصيفى ـ تحليلى به صورت مقطعى در سال

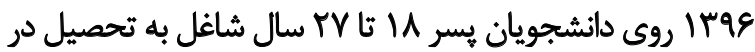

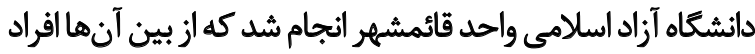

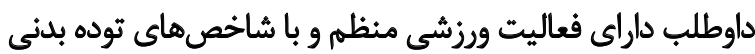

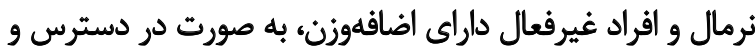

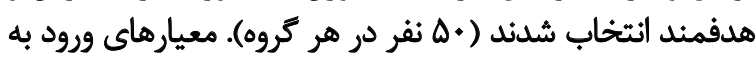

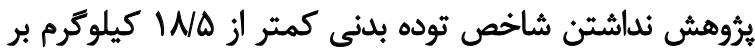


جدول ا. مقايسه ميائكين كيفيت خواب و ابعاد آن در بـسران دانشجو با و بدون فعاليت ورزشى منظم

\begin{tabular}{|c|c|c|c|c|}
\hline $\mathbf{P} *$ & $P^{*}$ & ميانكين+انحراف معيار & 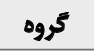 & ابعاد كيفيت خواب \\
\hline \multirow{2}{*}{$* / *+F$} & $<+1 .+1$ & $V / I T \pm \cdot / A r$ & غيرفعال & \multirow{2}{*}{ توصيف كلى فرد از خواب } \\
\hline & $<+1 \cdot+1$ & $.1 . \pm W /+A$ & ق فعال & \\
\hline \multirow{2}{*}{.$/ . T H \#$} & $<.1 . .1$ & $V / \Delta+ \pm \cdot / A r$ & غيرفعال & \multirow{2}{*}{ تأخير در به خواب رقتنن } \\
\hline & $<.1 . .1$ & $1 / \pm I T / V F$ & 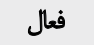 & \\
\hline \multirow{2}{*}{$.1 . .8 \#$} & $<.1 . .1$ & $1 / \pi T \pm \cdot / 99$ & تمعيرف & \multirow{2}{*}{ طول مدت خواب مفيد } \\
\hline & $<.1 .+1$ &. $\pm \pm Q Y / a r$ & فعال & \\
\hline \multirow{2}{*}{$\circ / . \% \Delta \#$} & $<+1++1$ & $+/ \Delta T \pm+/ T T$ & غيرفعال & \multirow{2}{*}{ كثايت خواب } \\
\hline & $<+1 \cdot+1$ & $.1 . \pm P E / T$. & 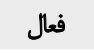 & \\
\hline \multirow{2}{*}{$.1 .4 \%$} & $<.1 . .1$ & $. / 9 \Delta \pm * / \Delta q$ & غ غيرفعال & \multirow{2}{*}{ أختالالات خواب } \\
\hline & $<+1+\infty 1$ & $+1+ \pm p q / *$ & 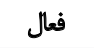 & \\
\hline \multirow{2}{*}{$<\bullet / .+1 \#$} & $<.1 . .1$ & $\cdot N \cdot \pm \cdot / \Delta r$ & غيرف فال & \multirow{2}{*}{ ميزان داروى خوابأور مصرفى } \\
\hline & $<.1 . .1$ &.$/ \pm \pm \varphi \cdot \pi$ & 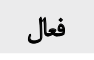 & \\
\hline \multirow{2}{*}{$<.1 . .1 \#$} & $<.1+.1$ & $1 / 9+ \pm+18 T$ & غيرفعال & \multirow{2}{*}{ اختالالات عملكرد دوزانه } \\
\hline & $<.1 .+1$ & $V / \pm \Delta \Delta / \& r$ & 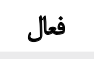 & \\
\hline \multirow{2}{*}{$<+/++1 \#$} & $\% 1 \%$ & $N \cdot \Delta \pm 1 / / r$ & ع غعيرفعال & \multirow{2}{*}{ نمره كيفيت كلى خواب } \\
\hline & $\%$ & $\Delta / / \pm \operatorname{YY} / A \Psi$ & 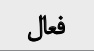 & \\
\hline
\end{tabular}

نرمافزار SPSS - S انجام شد.

تحقيق حاضر نيز ضريب آلفاى كرونباخ

از آزمونهاى كولموكروف اسميرنوف و لوين به ترثيب جهت

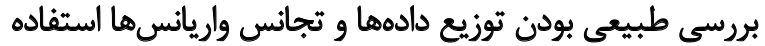

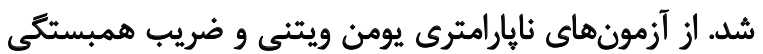

ميانكين و انحراف معيار قد و وزن و شاخص توده بدني

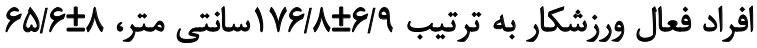

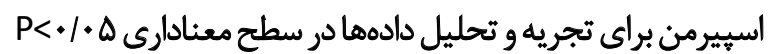

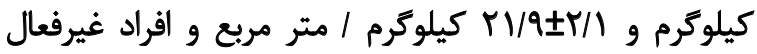

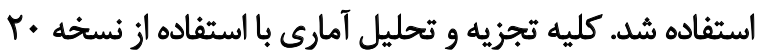

جدول r.ارتباط بين كيفيت خواب و ابعاد آن با شاخص توده بدن آزمودنى هاى تحقيق

\begin{tabular}{|c|c|c|c|c|c|c|}
\hline \multicolumn{6}{|c|}{ شاخص توده بدنى } & \multirow{3}{*}{ كيفيث كلي خواب } \\
\hline \multicolumn{3}{|c|}{ غيرفعال } & \multicolumn{2}{|c|}{ فعال } & \multirow{2}{*}{ ضريب r أزمييرمن آزمنها } & \\
\hline سطح معنى دارى & ضريب r اسييرمن & سطح معنى دارى & ضريب r اسييرمن & سطح معنى دارى & & \\
\hline . &.$- / 199$ & $\cdot 11 \cdot 1$ &.$/ M V$ & $.1+81$ &.$/ 719$ & توصيف كلى فرد از خواب \\
\hline.$/ \pi T$ &.$- / I F A$ &.$M r$ & $.1 . \Delta \Delta$ & $.1+A r$ &.$/ 190$ & تاخير در به خواب رقتن \\
\hline.$|89|$ & -.1 .80 & - NET & $-\rightarrow / T r \Delta$ & $.1 * A r$ & .112 & طول مدت خواب مفيد \\
\hline$\cdot / \Delta \Delta Y$ & $+1.9 V$ &.$/ \Delta \Delta T$ & $.1 .8 V$ &.$/ \Delta \Delta Y$ & $.1+97$ & كفايت خواب \\
\hline$\cdot 10 \cdot 1$ & $-\cdot 11 \cdot 1$ & .100 & $-.1 .9 Y$ & $.1 .10^{\circ}$ &.$/ T M$ & اختلالات خواب \\
\hline$\cdot M \cdot r$ &.$- / 19 V$ & $\cdot N \cdot r$ & $.1 .8 T$ & $\% . .1^{*}$ &.$/ T V \Delta$ & ميزان هاروى خوابآور هصرفى \\
\hline . MAF & . IOTT & $\cdot / 4 \cdot 1$ & ./NT & $.1+4 r^{*}$ &.$/ T A$ & الختالات عملكرد روزانه \\
\hline $.1+\pi$ &.$/ N \Delta \Delta$ &.$/ W$ & $\%$ & $\circ \ldots *$ &.$/ \pi T Y$ & نمره كيفيت كلى خواب \\
\hline
\end{tabular}


جوان رواج زيادى دارد و سبك زندگى كمتحرك مى تواند منجر

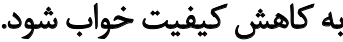

از جمله يافتههاي ديكر تحقيق حاضر وجود نمرات بإيينتر

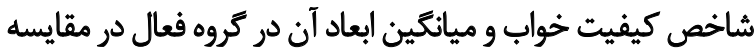

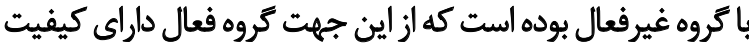

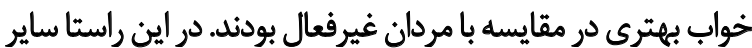

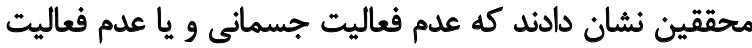

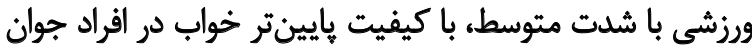

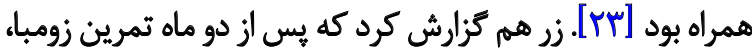

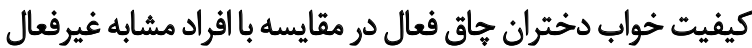

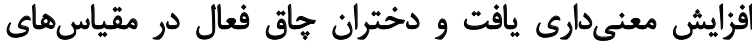

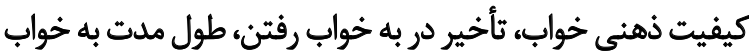

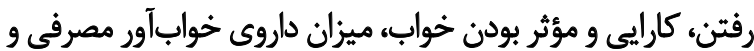

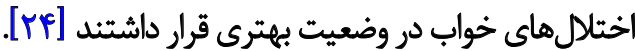
ابراهيمى تركمانى و همكاران ميانكين نمره كيفيت خواب

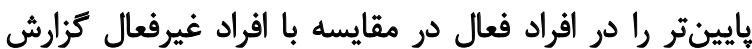

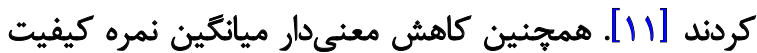

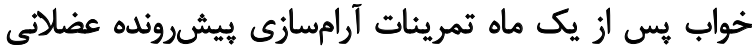

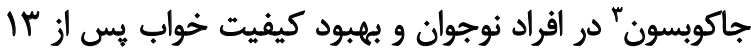

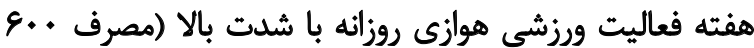

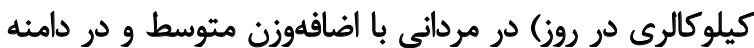

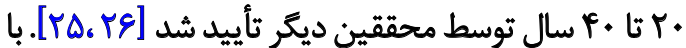

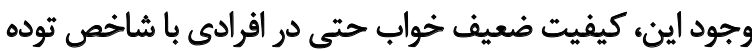

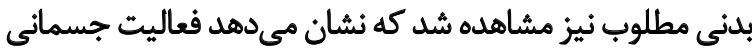

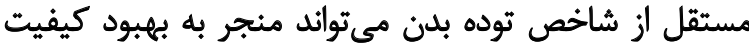
خواب در افراد جوان فعال شود [1] تئ.

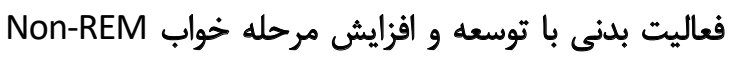

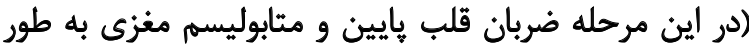

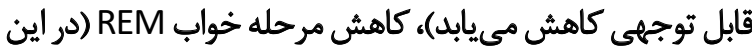

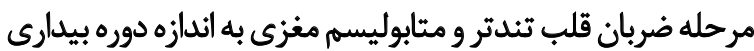

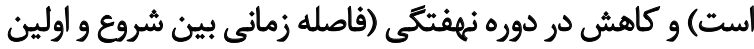

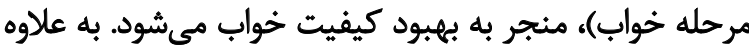

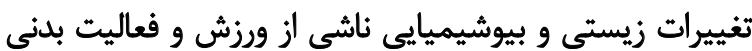

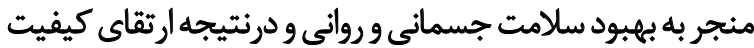

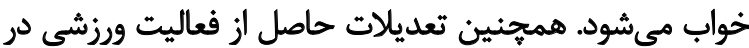

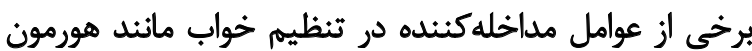

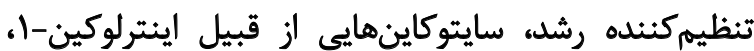

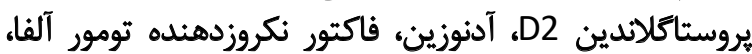

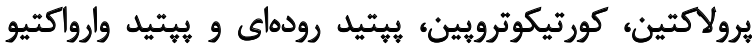

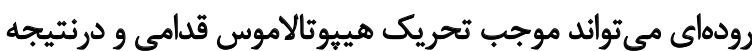

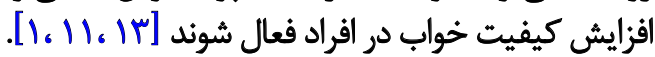

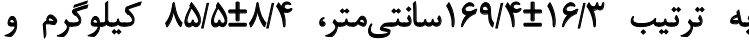

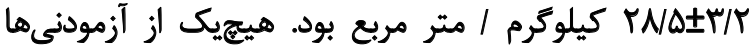

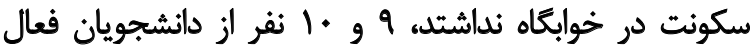

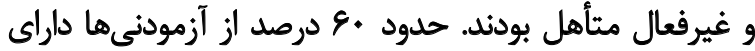

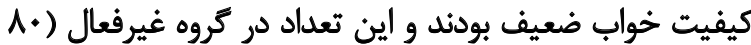

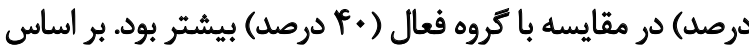

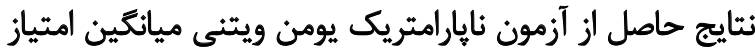

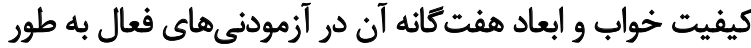

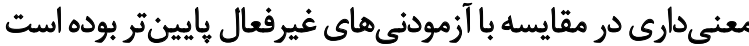

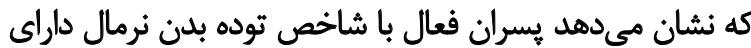

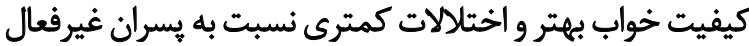

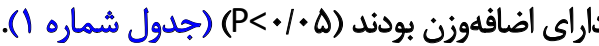
همجنين ارتباط مستقيم و معنى دارى بين نمره كيفيت خواب

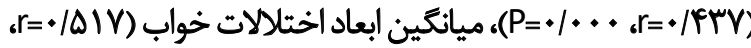

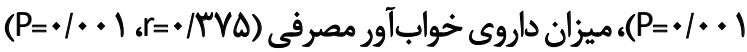

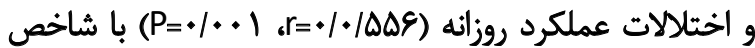

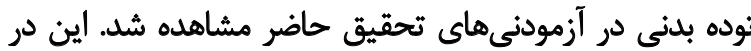

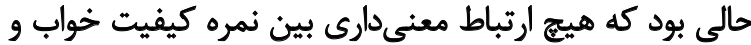

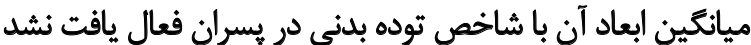

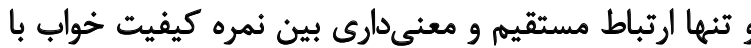
شاخص توده بدن يسران غيرفعال وجود داشت (جدول شماره بره).

\section{بحث و نتيجنه كيرى}

نتايج مطالعه حاضر نشان داد كه حدود .8 دروصد از

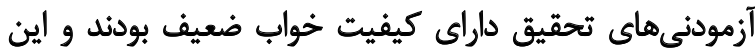

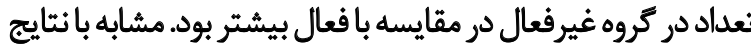

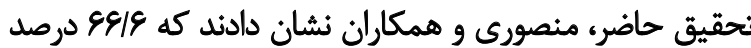

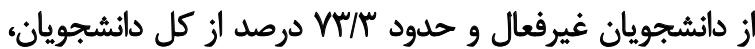

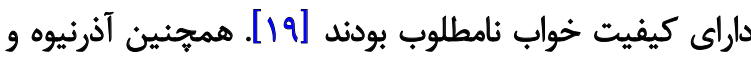

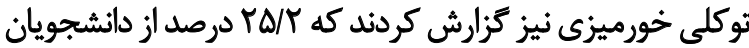

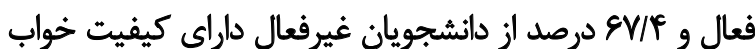

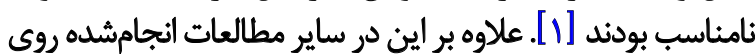

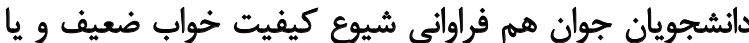

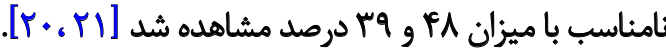

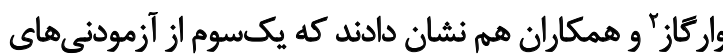

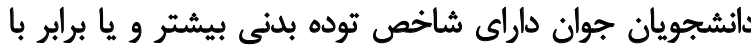

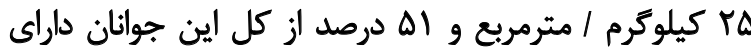

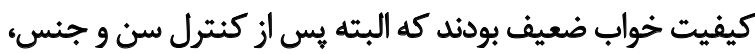

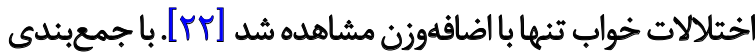

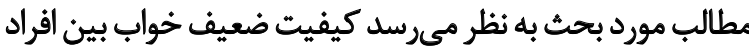




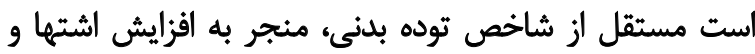

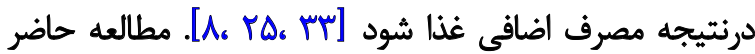

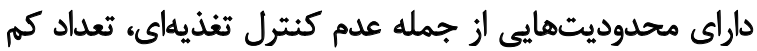

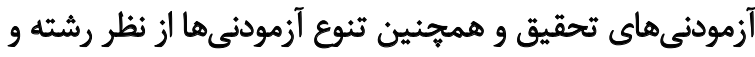

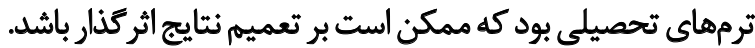

به طور خلاصه يافتههاى ثئوهش حاضر حاكى از شيوع زياد

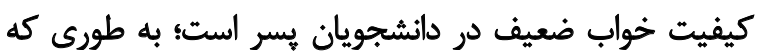

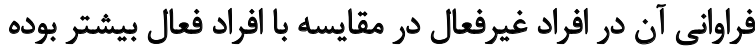

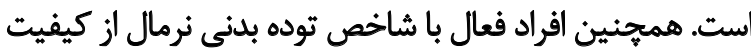

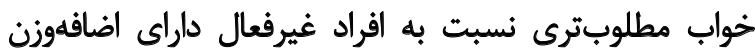

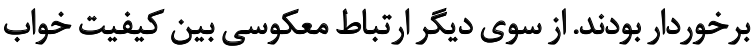

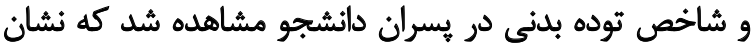

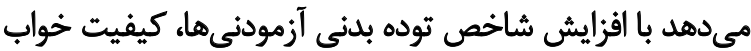

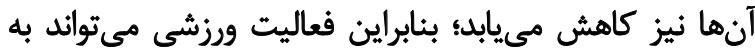

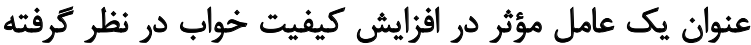

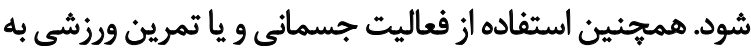
عنوان يك درمان غيردارويى براي بهبود كيفيت خواب بهاب به افرادي

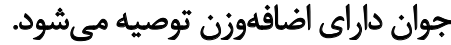

مالاحظاث الخالاقي

\section{يبيروى أز اصول اخلاق يُوهش}

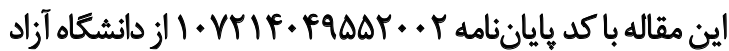

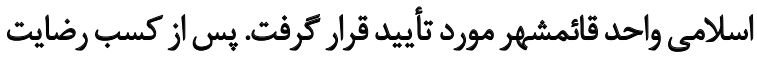

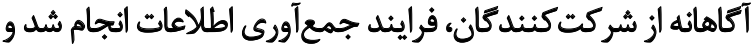

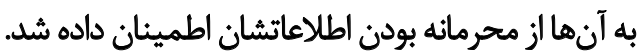

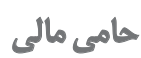

اين مقاله حامى مالى ندارد و بركرفته از يايان إمامه كارشناسى

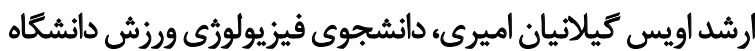

آزاد اسلامى واحد قائمشهر بود.

$$
\text { مشاركت نويسند مكان }
$$

تمام نويسندكان در طراحى، اجرا و نكًارش همه بخشهاى دارئ

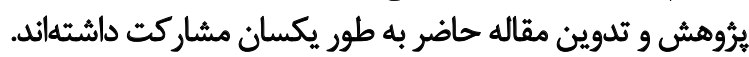

$$
\text { ت تعارض مثأع }
$$

بنابه اظهار نويسندكان، هيج تعارض منافع وجود ندارد.
از جمله يافتههاى ديكر تحقيق وجود ارتباط مستقيم و و

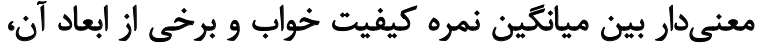

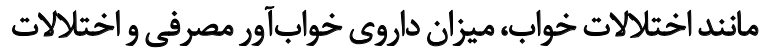

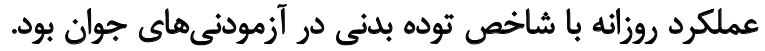

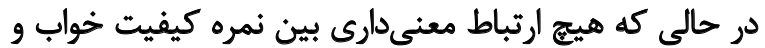

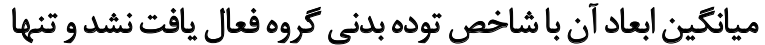

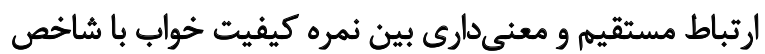

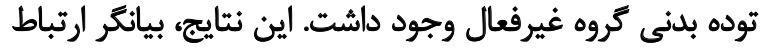

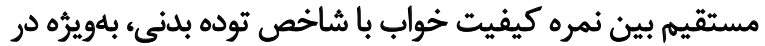

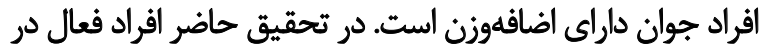

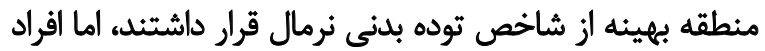

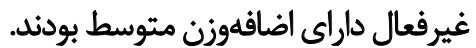
همسو با نتايج تحقيق حاضر ارتباط بين شاخص توده بدنى

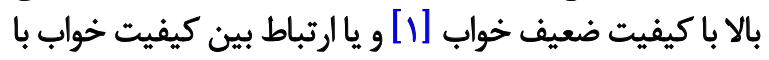

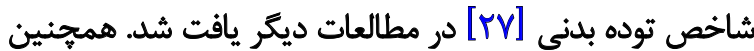

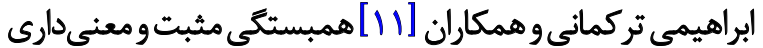

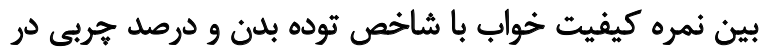

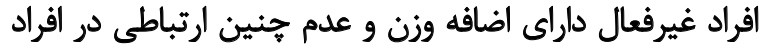

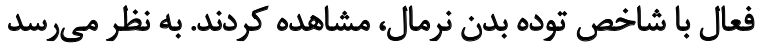

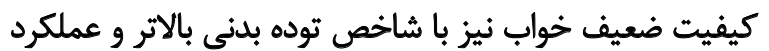

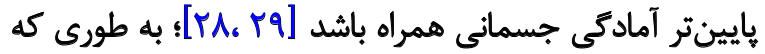

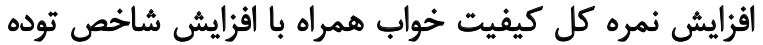

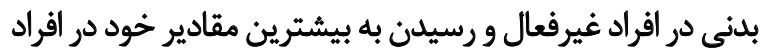

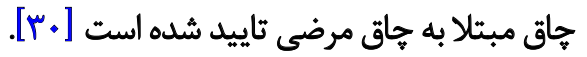
خواب در تنظيم وزن بدن نقش مهمى دارد. بـىخوابى ممكن

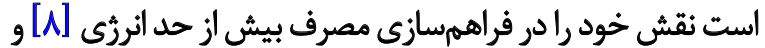

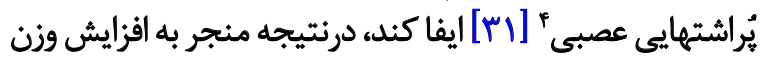
شود. افزايش مزمن كلوكوكورتيكوئيدها، مانند كورتيزول، مشابه

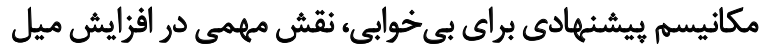

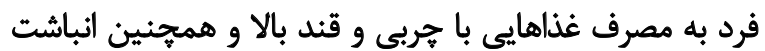

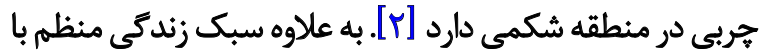

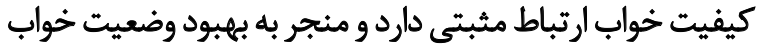

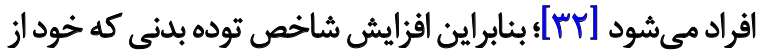

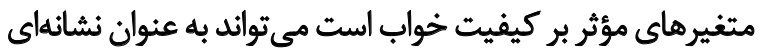

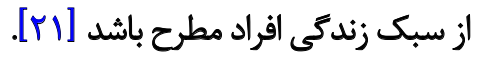

از اين جهت به نظر ميرسد كه هريك از متغيرهاى عدم شيوه

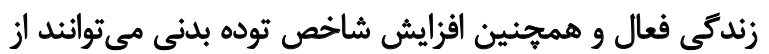

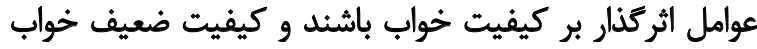

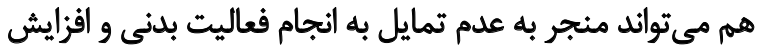

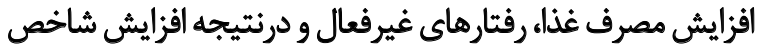

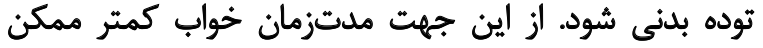




\section{References}

[1] Azarniveh MS, Tavakoli Khormizi SA. Effect of physical activity on quality of sleep in female students. J Gorgan Univ Med Sci. 2016; 18(2):108-14. [In Persian] http://goums.ac.ir/journal/ article-1-2770-fa.html

[2] Hargens TA, Kaleth AS, Edwards ES, Butner KL. Association between sleep disorders, obesity, and exercise: A review. Nat Sci Sleep. 2013; 5:27-35. [DOI:10.2147/NSS.S34838] [PMID] [PMCID]

[3] Murray K, Godbole S, Natarajan L, Full K, Hipp JA, Glanz K, et al. The relations between sleep, time of physical activity, and time outdoors among adult women. PLoS One. 2017; 12(9):e0182013. [DOI:10.1371/journal.pone.0182013] [PMID] [PMCID]

[4] Harvey AG, Stinson K, Whitaker KL, Moskovitz D, Virk H. The subjective meaning of sleep quality: A comparison of individuals with and without insomnia. Sleep. 2008; 31(3):383-93. [DOI:10.1093/sleep/31.3.383] [PMID] [PMCID]

[5] Ford ES, Li C, Wheaton AG, Chapman DP, Perry GS, Croft JB. Sleep duration and body mass index and waist circumference among U.S. adults. Obesity (Silver Spring). 2014; 22(2):598607. [DOI:10.1002/oby.20558] [PMID] [PMCID]

[6] Mehdizadeh S, Salari MM, Ebadi A, Abbasi S, Naderi Z, Sadeghi-Hasanabadi A. Relationship between sleep quality and body mass index in chemical warfare victims with bronchiolitis obliterans. J Qazvin Univ Med Sci. 2012; 16(1):29-35. [In Persian] http://journal.qums.ac.ir/article-1-1240-en.html

[7] Pearson NJ, Johnson LL, Nahin RL. Insomnia, trouble sleeping, and complementary and alternative medicine: Analysis of the 2002 national health interview survey data. Arch Intern Med. 2006; 166(16):1775-82. [DOI:10.1001/archinte.166.16.1775] [PMID]

[8] Taheri S. The link between short sleep duration and obesity: We should recommend more sleep to prevent obesity. Arch Dis Child. 2006; 91(11):881-4. [DOI:10.1136/adc.2005.093013] [PMID] [PMCID]

[9] Kondracki NL. The link between sleep and weight gain-research shows poor sleep quality raises obesity and chronic disease risk. Today's Dietitian. 2012; 14(6):48. https://www. todaysdietitian.com/newarchives/060112p48.shtml

[10] Vgontzas AN, Bixler EO, Lin HM, Prolo P, Mastorakos G, Vela-Bueno $A$, et al. Chronic insomnia is associated with nyctohemeral activation of the hypothalamic-pituitary-adrenal axis: Clinical implications. J Clin Endocrinol Metab. 2001; 86(8):3787-94. [DOI:10.1210/jcem.86.8.7778] [PMID]

[11] Ebrahemi-Torkmani B, Siahkouhian M, Azizkhahe-alanag A. The assessment of correlation between sleep quality and lung function indices and body mass index in active and inactive male students of Mohaghegh Ardabili University in 2017. J Rafsanjan Univ Med Sci. 2017; 16(8):743-56. [In Persian] http:// journal.rums.ac.ir/article-1-3852-en.html

[12] Passos GS, Poyares D, Santana MG, Garbuio SA, Tufik S, Mello MT. Effect of acute physical exercise on patients with chronic primary insomnia. J Clin Sleep Med. 2010; 6(3):270-5. [DOI:10.5664/jcsm.27825] [PMID] [PMCID]
[13] Veqar Z, Ejaz Hussain M. Sleep quality improvement and exercise: A review. Int J Sci Res Publ. 2012; 2(8):1-8. https:// www.researchgate.net/publication/236582394

[14] Rahmani Nia F, Mohebi H, Saberian M. The effect of walking on quality, quantity and some physiological parameters related to sleep in old men. J Sport Biosci. 2009; 1(3):111-26. [In Persian] https://jsb.ut.ac.ir/article_22017.html

[15] Bocicor AE, Buicu G, Sabau D, Varga A, Tilea I, Gabos-Grecu I. Association between sleep disorder and increased body mass index in adult patients. Acta Marisiensis. 2016; 62(2):221-4. [DOI:10.1515/amma-2016-0015]

[16] Hesar Koushki M, Mollanovruzi A, Hamedinia M. The prevalence of obesity and its related factors in 30-50 year old women of Neyshabur with an emphasis on physical activity. Iran J Endocrinol Metab. 2013; 14(5):472-8. [In Persian] http://ijem. sbmu.ac.ir/article-1-1370-en.html

[17] Tartibian B, Yaghoob Nezhad F, Abdollah Zadeh N. Comparison of respiratory parameters and sleep quality in active and none active young men: Relationship between respiratory parameters and sleep quality. Razi J Med Sci. 2014; 20(117):30-9. [In Persian] http://rjms.iums.ac.ir/article-1-2969-en.html

[18] Hasanzadeh M, Alavi Kariminejad Najmabadi K, Ghalehband MF, Yad Elahi Z, Gharaei B, Sadeghikia A. Sleep quality in Iranian drivers recognized as responsible for severe road accidents. J Res Behav Sci. 2008; 6(12):97-107. [In Persian] https://www. sid.ir/fa/journal/ViewPaper.aspx?ID=88962

[19] Mansouri A, Mokhayeri Y, Mohammadi Farrokhran E, Tavakkol Z, Fotouhi A. Sleep quality of students living in dormitories in Tehran University of Medical Sciences (TUMS) in 2011. Iran J Epidemiol. 2012; 8(2):71-82. [In Persian] http://irje.tums.ac.ir/ article-1-12-en.html

[20] Farhadi Nasab A, Azimi H. Study of patterns and subjective quality of sleep and their correlation with personality traits among Medical Students of Hamadan University of Medical Sciences. Avicenna J Clin Med. 2008; 15(1):11-5. [In Persian] http://sjh.umsha.ac.ir/article-1-378-en.html

[21] Rezaei Ardani A, Talaei A, Borhani Moghani M, Nejati R, Sabouri S, Solooti S, et al. Assessment the rules of demographic variables and body mass index in sleep quality among medical students. J Fundam Ment Health. 2012; 14(54):132-9. [In Persian] [DOI:10.22038/JFMH.2012.983]

[22] Vargas PA, Flores M, Robles E. Sleep quality and body mass index in college students: The role of sleep disturbances. J Am Coll Health. 2014; 62(8):534-41. [DOI:10.1080/07448481.201 4.933344] [PMID] [PMCID]

[23] Gerber M, Brand S, Holsboer-Trachsler E, Pühse U. Fitness and exercise as correlates of sleep complaints: Is it al in our minds? Med Sci Sports Exerc. 2010; 42(5):893-901. [DOI:10.1249/MSS.0b013e3181c0ea8c] [PMID]

[24] Zar A. Survey the effect of Zumba training on sleep quality in overweight girls. J Qazvin Univ Med Sci. 2017; 21(1):45-52. [In Persian] http://journal.qums.ac.ir/article-1-2252-en.html

[25] Roozbahani T, Norian M, Saatchi K, Moslemi A. Effects of progressive muscle relaxation on sleep quality in pre-university stu- 
dents: A randomized clinical trial. Sci J Hamadan Nurs Midwifery Fac. 2016; 24(1):16-23. [In Persian] [DOI:10.20286/nmj-24013]

[26] Kjeldsen JS, Rosenkilde $M$, Nielsen SW, Reichkendler $M$, Auerbach P, Ploug T, et al. Effect of different doses of exercise on sleep duration, sleep efficiency and sleep quality in sedentary, overweight men. Bioenergetics. 2012; 2(1):1-6. [DOI:10.4172/2167-7662.1000108]

[27] Madrid-Valero JJ, Martínez-Selva JM, Ordoñana JR. Sleep quality and body mass index: A co-twin study. J Sleep Res. 2017; 26(4):461-7. [DOI:10.1111/jsr.12493] [PMID]

[28] Chang SP, Chen YH. Relationships between sleep quality, physical fitness and body mass index in college freshmen. J Sports Med Phys Fitness. 2015; 55(10):1234-41. [PMID]

[29] Ferranti R, Marventano S, Castellano S, Giogianni G, NoIfo $F$, Rametta $S$, et al. Sleep quality and duration is related with diet and obesity in young adolescent living in Sicily, Southern Italy. Sleep Sci. 2016; 9(2):117-22. [DOI:10.1016/j. slsci.2016.04.003] [PMID] [PMCID]

[30] Ozgul Tepe S, Gunes G, Pehlivan E, Genc M. The relationship between body mass index and Pittsburgh sleep quality index: Erkan Pehlivan. Eur J Public Health. 2017; 27(Suppl 3):ckx189.173. [DOI:10.1093/eurpub/ckx189.173]

[31] Jalilolghadr Sh, Pakpour-Hajiagha A, Yousefi A, Pakzad R. Association of sleep habits with dietary pattern; a schools-based cross-sectional study. J Qazvin Univ Med Sci. 2018; 22(3):2331. [In Persian] [DOI:10.29252/qums.22.3.23]

[32] Lima PF, Medeiros ALD, Araujo JF. Sleep-wake pattern of medical students: Early versus late class starting time. Braz J Med Biol Res. 2002; 35(11):1373-7. [DOI:10.1590/S0100879X2002001100016] [PMID]

[33] Gupta NK, Mueller WH, Chan W, Meininger JC. Is obesity associated with poor sleep quality in adolescents? Am J Hum Biol. 2002; 14(6):762-8. [DOI:10.1002/ajhb.10093] [PMID] 